\title{
Prognostic Value of Cumulative Score Based on Preoperative Fibrinogen and Albumin Level in Skull Base Chordoma
}

\author{
Mingxuan $\mathrm{Li}^{1}$ \\ Jiwei $\mathrm{Bai}^{2}$ \\ Shuai Wang' \\ Yixuan Zhai ${ }^{3}$ \\ Shuheng Zhang' \\ Chuzhong $\mathrm{Li}^{1}$ \\ Jiang $\mathrm{Du}^{4}$ \\ Yazhuo Zhang (D) ${ }^{1,2,5-7}$
}

'Beijing Neurosurgical Institute, Capital Medical University, Beijing, People's Republic of China; ${ }^{2}$ Department of Neurosurgery, Beijing Tiantan Hospital, Capital Medical University, Beijing,

People's Republic of China; ${ }^{3}$ Department of Neurosurgery, The First Affiliated Hospital of Zhengzhou University, Zhengzhou, People's Republic of China; ${ }^{4}$ Department of Neuropathology, Beijing Neurosurgical Institute, Capital Medical University, Beijing, People's Republic of China; ${ }^{5}$ Beijing Institute for Brain Disorders Brain Tumor Center, Beijing, People's Republic of China; ${ }^{6}$ China National Clinical Research Center for Neurological Diseases, Beijing, People's Republic of China; ${ }^{7}$ Key Laboratory of Central Nervous System Injury Research, Capital Medical University, Beijing,

People's Republic of China
Correspondence: Yazhuo Zhang Email zyz2004520@yeah.net
This article was published in the following Dove Press journal: OncoTargets and Therapy

\begin{abstract}
Objective: Inflammation and malnutrition have been shown to be correlated with tumor progression and a poor prognosis in various cancers. However, the clinical implications of biomarkers of inflammation and malnutrition in chordoma have not been elucidated. We attempted to characterize the fibrinogen and albumin levels in skull base chordoma and investigate their correlations with clinicopathological data and survival.
\end{abstract}

Methods: The preoperative levels of fibrinogen and albumin were assessed in 183 primary skull base chordoma patients. The cutoff values were determined by X-tile software, and their correlations with patient prognosis were further explored using the Kaplan-Meier curve and Cox proportional hazards regression analysis. In addition, the predictive performances of these markers in survival were evaluated by receiver operating characteristic curves.

Results: The values of fibrinogen and albumin in skull base chordoma patients ranged from 1.73 to 7.40 and 37.6 to $53.0 \mathrm{~g} / \mathrm{L}$, respectively. The optimal cutoff values for fibrinogen and albumin were 3.29 and $44.60 \mathrm{~g} / \mathrm{L}$, respectively. Fibrinogen and albumin were correlated with the patient age and tumor pathology types. Albumin, but not fibrinogen, was associated with the patients' progression-free survival and overall survival. Importantly, the FA score, which combines fibrinogen and albumin, could independently predict both progression-free survival and overall survival, and enhanced the performance of fibrinogen or albumin in survival prediction in skull base chordoma.

Conclusion: Our data reveal the clinical prognostic role of albumin and suggest that the FA score may be a valuable prognostic grading system in skull base chordoma.

Keywords: skull base chordoma, fibrinogen, albumin, biomarker, prognosis

\section{Introduction}

Skull base chordoma is a rare malignancy of the central nervous system originating from residual notochord. ${ }^{1}$ Radical surgery and postoperative radiotherapy remain the best treatment for increasing the life span of skull base chordoma patients. ${ }^{2}$ However, because skull base chordoma is adjacent to vital neurovascular tissues, is resistant to conventional radiotherapy and chemotherapy and has a high recurrence rate, the therapy of skull base chordoma has been a difficult challenge in neurosurgery. ${ }^{3-5} \mathrm{~A}$ risk stratification assessment of recurrence and death before therapy could help clinicians identify the optimal choice of treatment and improve the prognosis of patients. However, a dependable preoperative prognostic grading system that can accurately predict the individual clinical outcome of patients is currently lacking. 
An increasing number of studies have demonstrated that systematic inflammation and the nutritional status play significant roles in the tumor initiation, progression and clinical outcomes in various human cancers. ${ }^{6-8}$ In particular, fibrinogen, which is a type of glycoprotein produced by hepatic epithelium cells that is indispensable for the coagulation cascade, plays an important role in the human response to systematic inflammation and may reflect the inflammation status. ${ }^{9}$ Moreover, recent studies show that high levels of preoperative fibrinogen predict a poor prognosis in non-small cell lung cancer, high-grade glioma, colorectal cancer and renal cell carcinoma. ${ }^{10-13}$

Many studies have shown that malnutrition is closely associated with tumor progression and human immunity and that albumin, which is an acute-phase protein mostly synthesized by the liver and a nutritional indicator regularly used to evaluate the nutritional status, is involved in systemic inflammation. ${ }^{14,15}$ Moreover, low levels of serum albumin have been observed in several cancers compared to healthy controls. ${ }^{16}$ Several studies report that a low preoperative albumin level can represent an adverse prognostic factor for patient outcomes and a latent risk factor for postoperative complications in human cancers. ${ }^{17,18}$ Furthermore, considering the prognostic role of fibrinogen and albumin, the FA score, which is a novel scoring system that combines fibrinogen and albumin, has recently been reported, and this score can independently predict outcomes in patients with esophageal cancer and urothelial carcinoma. ${ }^{19,20}$

To the best of our knowledge, thus far, studies evaluating the prognostic performance of preoperative fibrinogen and/or albumin in skull base chordoma are limited. Therefore, in the current study, we retrospectively characterized the preoperative level of fibrinogen and albumin and investigated the association among fibrinogen, albumin, clinicopathological data and survival in 183 primary skull base chordoma patients who underwent surgery. In addition, the FA score ( 0 to 2 ) was generated and the prognostic role of the FA score was further analysed. We also compared the predictive value of fibrinogen, albumin and the FA score in skull base chordoma.

\section{Materials and Methods}

\section{Patients}

One hundred eighty-three eligible patients with primary skull base chordoma treated with surgical therapy at Beijing Tiantan Hospital, Capital Medical University, between January 2008 and September 2014 were retrospectively included in the current research. The inclusion criteria in this study were as follows: (a) histologically newly confirmed skull base chordoma; (b) first surgery with no preoperative radiotherapy and/or chemotherapy; (c) patients with complete clinicopathological information and preoperative level of fibrinogen and albumin; and (d) patients with follow-up data. Otherwise, patients were excluded for the following conditions: (a) unclear pathological diagnosis; (b) patients who received a previous treatment such as surgery, radiotherapy or chemotherapy; (c) patients with clinical evidence of chronic or acute infection/inflammation disease, haematological disease or a previous history of other malignancies; (d) without adequate clinical information and/or blood tests; and (e) lost to follow-up.

\section{Data Collection}

Basic clinical and laboratory information including age, gender, body mass index $\left(\mathrm{kg} / \mathrm{m}^{2}\right)$, clinical symptoms, histological type (classical chordoma, chondroid chordoma or dedifferentiated chordoma), ${ }^{4}$ tumor size $\left(\mathrm{mm}^{3}\right)$, brainstem involvement, tumor blood supply (abundant or others), tumor texture (soft or others), extent of resection (total resection: no evidence of residual tumor on postoperative magnetic resonance imaging; and non total resection), ${ }^{21}$ and preoperative fibrinogen $(\mathrm{g} / \mathrm{L})$ and albumin $(\mathrm{g} / \mathrm{L})$ levels were gathered from the medical record system.

\section{FA Score}

The optimal cutoff values for albumin and fibrinogen were defined as the values that generate the minimum $p$ value via a Log rank test between the groups in the overall survival analysis using X-tile 3.61 software (Yale University, New Haven, CT, USA). ${ }^{22}$ After identifying these values, the two original continuous variables were divided into categorical variables representing high and low levels. Then, we defined the FA score using a combination of fibrinogen and albumin as follows: score 2, patients with increased fibrinogen and decreased albumin; score 1, patients with either increased fibrinogen or decreased albumin; and score 0 , patients with neither increased fibrinogen or decreased albumin. ${ }^{19}$

\section{Follow-Up}

All patients were regularly followed up via outpatient examinations, e-mail and telephone, and the deadline of our follow-up period was October 2019. Tumor recurrence was diagnosed by evidence of new clinical symptoms and/or imaging findings. Progression-free survival (PFS), the 
interval between tumor resection to the firstly tumor recurrence, and overall survival (OS), the time from the date of tumor surgery to death, were identified for patient outcome assessment. For those who were progression-free (PFS) or alive (OS) at the final follow-up, the observations were censored.

\section{Statistical Analysis}

All analyses were conducted using IBM SPSS, version 19.0 (IBM Corp., Armonk, NY, USA) and GraphPad prism 7.0 (GraphPad, La Jolla, CA, USA). The quantitative data are shown as the mean \pm standard deviation (SD), and the associations between categorical factors were analysed by the chi-square test or Wilcoxon rank sum test. The KaplanMeier method and Log rank test were performed for the PFS and OS analysis. Multivariable Cox proportional hazard regression models that included variables with $p<0.10$ in the univariable analysis were applied to determine the independent variables affecting PFS and OS. The abilities of fibrinogen, albumin and the FA score to predict PFS and OS were compared by receiver operating characteristic (ROC) analysis. All tests were 2 -sided, and a $p$ value $<0.05$ was considered statistically significant.

\section{Results}

\section{Patients Characteristics}

In the current study, 183 skull base chordoma patients were included between January 2008 and September 2014 following inclusive criteria. Of these patients, ninety six patients were men, and 87 patients were women, and the mean age at diagnosis was 40.1 (SD, 15.2) years. The most common symptom was headache $(85 / 183)$, followed by diplopia $(66 /$ $183)$ and blurred vision (59/183). The classical chordoma histopathological subtype was found in 125 patients, and the remaining 58 patients were pathologically diagnosed with chondroid chordoma. The median tumor volume, which was calculated as half of the product of the longest diameters of the coronal, sagittal and axial axes, was $21,000.0 \mathrm{~mm}^{3}$. Patients were followed up until October 2019, and the median followup duration was 74 months. The clinicopathological data of the study cohort are described in detail in Table 1.

\section{Description of the Fibrinogen and Albumin Levels in Skull Base Chordoma Patients}

The level of fibrinogen ranged from 1.73 to $7.40 \mathrm{~g} / \mathrm{L}$ (median, $2.93 \mathrm{~g} / \mathrm{L}$ ) and the median serum albumin level was $45.8 \mathrm{~g} / \mathrm{L}$ (range, 37.6-53.0 g/L) in 183 skull base chordoma patients (Table 1). Then, we used X-tile software to assess the optimal cut off values, which were 3.29 for fibrinogen (Figure S1) and $44.60 \mathrm{~g} / \mathrm{L}$ for albumin (Figure S2). Of the 183 patients, 130 (71.0\%) patients had a fibrinogen level $<3.29 \mathrm{~g} / \mathrm{L}$ and an albumin level $<44.60 \mathrm{~g} / \mathrm{L}$ was found in $55(30.1 \%)$ patients. We further combined the two variables using the FA score as described above. Specifically, 93 (50.8\%) patients had an FA score of $0,72(39.3 \%)$ patients had an FA score of 1 and the remaining 18 patients $(9.8 \%)$ had an FA score of 2 .

\section{Association Among Fibrinogen, Albumin, the FA Score and the Clinicopathological Variables}

A higher fibrinogen level was correlated with an older age $(p<0.001)$ and a larger tumor volume $(p=0.011)$. Additionally, a higher fibrinogen level was observed in the classical chordoma type $(p=0.042)$ (Table 2$)$. In contrast, the albumin level was negatively correlated with patients' age $(p=0.022)$, and patients with chondroid chordoma had a higher albumin level $(p=0.026)$. Moreover, a lower level of albumin was associated with a soft tumor texture $(p=0.023)$ (Table 2). Strong associations were observed between a higher FA score and an older age $(p<0.001)$ and the classical chordoma pathological type $(p=0.005)$. Tumors with a soft texture tended to have a lower FA score, although the correlation was not statistically significant $(p=0.122)$. No statistically significant associations were identified between the FA score and other clinical variables, including sex, tumor volume, blood supply and brainstem involvement (Table 2).

\section{Analysis of the Association Between Fibrinogen, Albumin or the FA Score and Patients' Survival}

At the final follow-up, 128 (69.9\%) patients experienced tumor progression and $72(39.3 \%)$ patients died. The follow-up time ranged from 3 to 141 months (median 74 months), and the median PFS and OS were 30 months and 74 months, respectively. The Kaplan-Meier curve indicated that the median PFS (Figure 1A) and OS (Figure 1B) of the patients with high fibrinogen were shorter than those in the low fibrinogen group (PFS: 25 months vs 36 months; OS: 96 months vs 125 months), although the differences were not significant $(p=0.056$, $0.059, \log$ rank test, respectively). In contrast, the patients 
Table I Summary of Clinicopathological Features in 183 Skull Base Chordoma Patients

\begin{tabular}{|c|c|}
\hline Characteristic & Number of Patients, (\%) \\
\hline Median age (year, mean $\pm S D$ ) & $4 I .0(40.1 \pm 15.2)$ \\
\hline \multicolumn{2}{|l|}{ Sex } \\
\hline Male & $96(52.5)$ \\
\hline Female & $87(47.5)$ \\
\hline Median tumor volume & $21,000.0$ \\
\hline$\left(\mathrm{mm}^{3}\right.$, mean $\left.\pm \mathrm{SD}\right)$ & $(31,700.9 \pm 33,3 \mid 8.2)$ \\
\hline \multicolumn{2}{|l|}{ Symptom } \\
\hline Headache & $85(46.4)$ \\
\hline Diplopia & $66(36.1)$ \\
\hline Blurred vision & $59(32.2)$ \\
\hline Vision field defect & $42(23.0)$ \\
\hline Dizziness & $30(16.4)$ \\
\hline \multicolumn{2}{|l|}{ Tumor texture } \\
\hline Soft & $55(30.1)$ \\
\hline Others (hard or moderate) & $128(69.9)$ \\
\hline \multicolumn{2}{|l|}{ Tumor blood supply } \\
\hline Rich & $107(58.5)$ \\
\hline Others (poor or moderate) & $76(4 \mid .5)$ \\
\hline \multicolumn{2}{|l|}{ Pathology type } \\
\hline Classical & $125(68.3)$ \\
\hline Chondroid & $58(31.7)$ \\
\hline Dedifferentiated & $0(0)$ \\
\hline \multicolumn{2}{|l|}{ Brainstem involvement } \\
\hline No & $68(37.2)$ \\
\hline Yes & $115(62.8)$ \\
\hline $\begin{array}{l}\text { Median body mass index } \\
\left(\mathrm{kg} / \mathrm{m}^{2}, \text { mean } \pm \mathrm{SD}\right)\end{array}$ & $23.0(23.3 \pm 3.2)$ \\
\hline Median fibrinogen $(g / L$, mean $\pm S D)$ & $2.93(3.00 \pm 0.73)$ \\
\hline Median albumin $(g / L$, mean $\pm S D)$ & $45.8(46.0 \pm 3.1)$ \\
\hline Recurrence during follow-up & $128(69.9)$ \\
\hline Death during follow-up & $72(39.3)$ \\
\hline
\end{tabular}

Abbreviation: SD, standard deviation.

with a high level of albumin had longer PFS than the patients with an albumin level $<44.60 \mathrm{~g} / \mathrm{L}$ (median PFS time: 36 months vs 21 months, $p=0.024$, Log rank test) (Figure 1C). Furthermore, the median OS in the patients with high albumin was 125 months, which was significantly longer than that in the low-albumin patients (median OS, 72 months, $p<0.001$ ) (Figure 1D). A higher FA score was associated with worse PFS ( $p=0.014$, Figure 1E) and OS $(p<0.001$, Figure 1F) according to the KaplanMeier analysis. In particular, significant differences in PFS were observed between the patients with an FA score of 0 and those with an FA score of 1 (median PFS time: 42 months vs 24 months, $p=0.012$, Log rank test) or FA score of 2 (median PFS time: 42 months vs 21 months, $p=0.026$, Log rank test). Regarding OS, the Log rank tests showed significant differences in OS between the FA score 2 group and FA score 1 group (median time: 47 months vs 100 months, $p=0.011$ ) and FA score 0 group (median time: 47 months vs 125 months, $p<0.001$ ).

We further performed a subgroup analysis of the FA score with different pathology types and tumor volume chordoma. No significant difference in PFS was observed among the different FA score groups in the classical chordoma patients $(p=0.205)$ (Figure 2A). However, a high FA score was associated with a shorter PFS in the chondroid chordoma patients $(p=0.041)$ (Figure $2 \mathrm{~B})$. Moreover, the classical chordoma patients in the high FA score group had a shorter OS ( $p=0.001$ ) (Figure 2C), but this difference was not significant among the chondroid chordoma patients $(p=0.527)$ (Figure 2D). A high FA score was still correlated with poor OS among the patients with either tumor volume either $\leq 20,000 \mathrm{~mm}^{3}(p=0.005)$ or $>20,000 \mathrm{~mm}^{3}(p=0.007)$, although the differences in PFS were not significant $(p=0.185,0.242$, respectively) (Figure $2 \mathrm{E}-\mathrm{H}$ ).

We also performed a Cox survival analysis, and the results indicated that age $(p=0.018)$, tumor volume $(p=0.004)$, tumor texture $(p=0.013)$, blood supply $(p=0.045)$, pathology type $(p=0.031)$, degree of resection $(p<0.001)$, albumin level $(p=0.027)$ and FA score $(p=0.017)$, but not the fibrinogen level $(p=0.061)$, were associated with PFS in the univariable analysis. The multivariable analysis showed that the FA score $(p=0.026)$ was an independent predictor of PFS in skull base chordoma (Table 3). Additionally, we analysed the role of fibrinogen, albumin and the FA score in OS. A low albumin level $(p=0.001)$ and a high FA score $(p<0.001)$ were correlated with a shorter OS, and the FA score $(p<0.001)$ was identified as an independent prognostic indicator of OS (Table 4).

\section{Value of Fibrinogen, Albumin and the FA Score in Predicting Patient Outcomes}

Next, we compared the ability of fibrinogen, albumin and the FA score to predict patients' survival using ROC curve analysis. The ROC analysis showed that the FA score had more predictive power than fibrinogen or albumin in predicting 3-year (AUC, area under the curve: 0.609, 0.585 and 0.550 , respectively) and 5-year (AUC: $0.643,0.586$ and 0.594 , respectively) PFS (Figure 3A). Similar results were 
Table 2 Association Between Fibrinogen, Albumin, FA Score and Clinicopathological Features in Skull Base Chordoma

\begin{tabular}{|c|c|c|c|c|c|c|c|c|c|c|c|}
\hline \multirow[t]{2}{*}{ Variables } & \multirow[b]{2}{*}{$\mathbf{N}$} & \multicolumn{3}{|c|}{ Fibrinogen (g/L) } & \multicolumn{3}{|c|}{ Albumin (g/L) } & \multicolumn{4}{|c|}{ FA Score } \\
\hline & & $<3.29$ & $\geq 3.29$ & $P$ value & $<44.60$ & $\geq 44.60$ & $P$ value & 0 & $\mathbf{I}$ & 2 & $P$ value \\
\hline Sex & & & & 0.696 & & & 0.117 & & & & 0.632 \\
\hline Male & 96 & 67 & 29 & & 24 & 72 & & 52 & 35 & 9 & \\
\hline Female & 87 & 63 & 24 & & 31 & 56 & & 41 & 37 & 9 & \\
\hline Age & & & & $<0.00$ I* & & & $0.022^{*}$ & & & & $<0.001 *$ \\
\hline$\leq 55$ & $|5|$ & 117 & 34 & & 40 & 111 & & 88 & 52 & 11 & \\
\hline$>55$ & 32 & 13 & 19 & & 15 & 17 & & 5 & 20 & 7 & \\
\hline Tumor volume & & & & $0.011^{*}$ & & & 0.935 & & & & 0.222 \\
\hline$\leq 20,000 \mathrm{~mm} 3$ & 89 & 71 & 18 & & 27 & 62 & & 51 & 31 & 7 & \\
\hline$>20,000 \mathrm{~mm} 3$ & 94 & 59 & 35 & & 28 & 66 & & 42 & 41 & 11 & \\
\hline Texture & & & & 0.703 & & & $0.023^{*}$ & & & & 0.122 \\
\hline Soft & 55 & 38 & 17 & & 23 & 32 & & 24 & 22 & 9 & \\
\hline Others (hard or moderate) & 128 & 92 & 36 & & 32 & 96 & & 69 & 50 & 9 & \\
\hline Blood supply & & & & 0.319 & & & 0.547 & & & & 0.438 \\
\hline Abundant & 107 & 73 & 34 & & 34 & 73 & & 52 & 42 & 13 & \\
\hline Others (poor or moderate) & 76 & 57 & 19 & & 21 & 55 & & 41 & 30 & 5 & \\
\hline Pathology & & & & $0.042^{*}$ & & & $0.026 *$ & & & & $0.005^{*}$ \\
\hline Classical & 125 & 83 & 42 & & 44 & 81 & & 57 & 50 & 18 & \\
\hline Chondroid & 58 & 47 & 11 & & 11 & 47 & & 36 & 22 & 0 & \\
\hline Brainstem involvement & & & & 0.437 & & & 0.851 & & & & 0.178 \\
\hline No & 68 & 46 & 22 & & 21 & 47 & & 35 & 23 & 10 & \\
\hline Yes & 115 & 84 & 31 & & 34 & 81 & & 58 & 49 & 8 & \\
\hline Total & 183 & 130 & 53 & & 55 & 128 & & 93 & 72 & 18 & \\
\hline
\end{tabular}

Note: *Indicate $p<0.05$.

also found in the OS analysis as follows: the AUC of the FA score in the 3-year and 5-year OS analysis were 0.594 and 0.639 , which were larger than those of fibrinogen $(0.559$ and 0.574 ) and albumin (0.572 and 0.616) (Figure 3B).

\section{Discussion}

In the present study, for the first time, we characterized the level of fibrinogen and albumin, and investigated theirs associations with clinicopathological data in primary skull base chordoma patients. We found that the fibrinogen level and albumin level were correlated with age and the pathological types, which are potential risk factors for a poor outcome in chordoma patients. ${ }^{4,23}$ Low levels of albumin were associated with a poor PFS and OS. Moreover, the combined analysis of fibrinogen and albumin revealed that the FA score was an independent prognostic factor for PFS and OS. Additionally, the FA score showed better predictive ability than fibrinogen or albumin alone; the patients with an FA score of 2 (high fibrinogen and low albumin) had the worst prognosis. Our findings reveal that the FA score may be a potential marker for screening high-risk groups and that patients with an FA score of 2 should be more closely monitored. The FA score can be easily acquired from a routine blood test and may help clinicians evaluate personal prognosis. Agents targeting fibrinogenmediated inflammation and albumin supplements may provide a valuable complementary therapy for the treatment of chordoma patients.

Increasingly, studies show that systemic inflammation and malnutrition play essential roles in tumor progression, therapy resistance and survival of patients with malignancies. ${ }^{6,24,25}$ The identification of preoperative laboratory biomarkers predicting patient outcomes before treatment is of great importance, possibly enabling the guidance of risk stratification and more appropriate allocation of therapeutic schedules. However, to date, knowledge of inflammatory and nutritional prognostic biomarkers in chordoma is limited. Fibrinogen has been considered an important regulator and biomarker of 


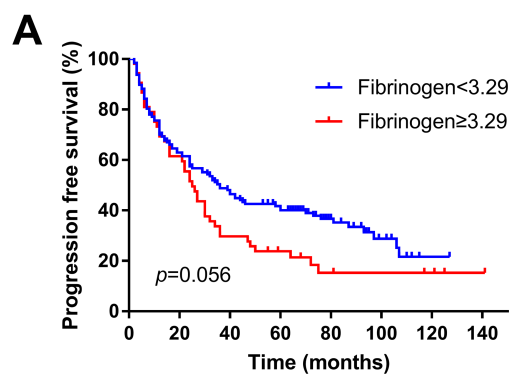

D

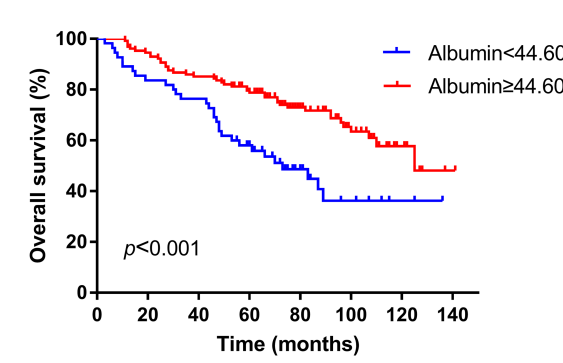

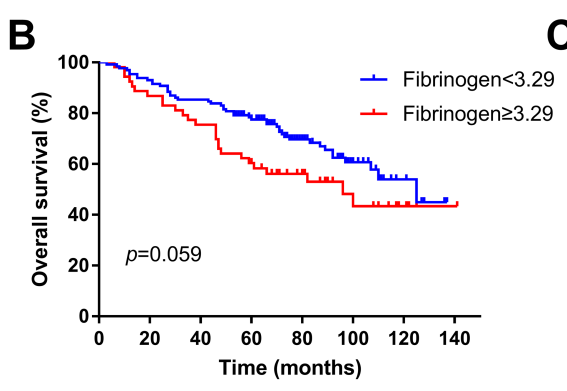

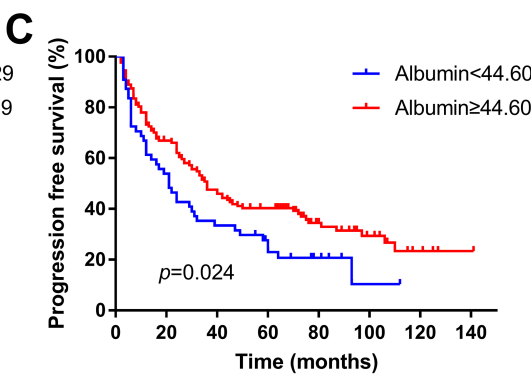

E

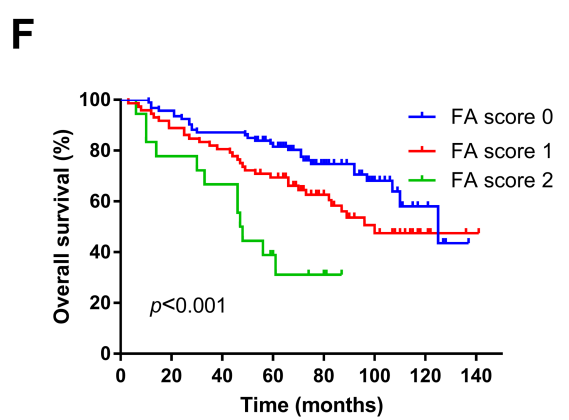

Figure I Kaplan-Meier analysis of fibrinogen, albumin and FA score in skull base chordoma. (A) Fibrinogen and PFS. (B) Fibrinogen and OS. (C) Albumin and PFS. (D) Albumin and OS. (E) FA score and PFS. (F) FA score and OS.

Abbreviations: PFS, progression-free survival; OS, overall survival.
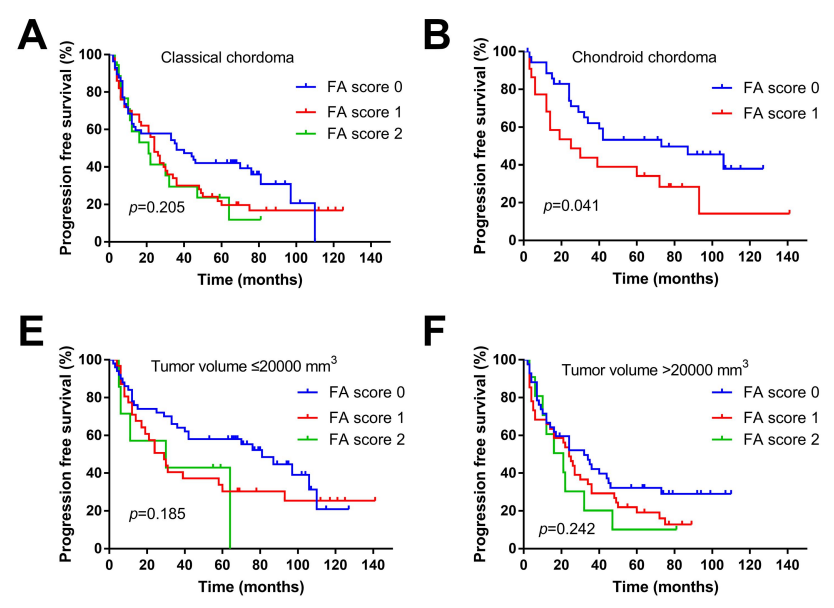

$\mathbf{F}$

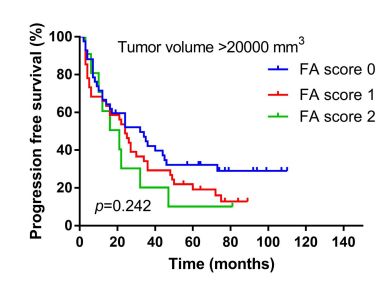

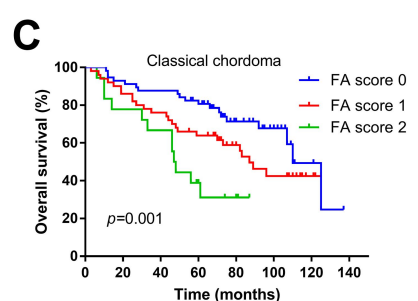

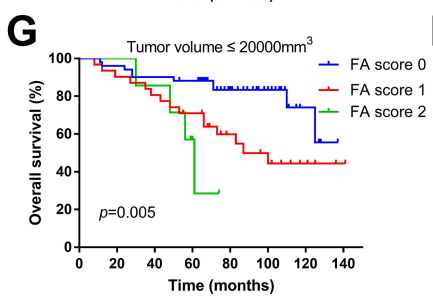

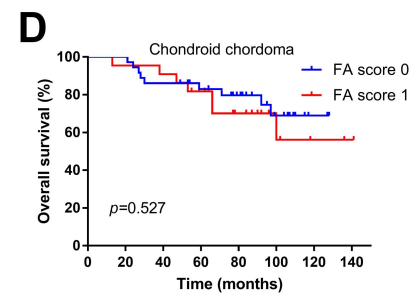

$\mathrm{H}$

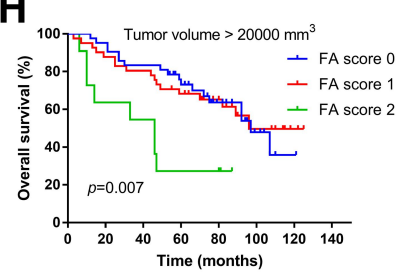

Figure 2 Kaplan-Meier analysis of FA score in different subgroups of skull base chordoma patients. (A) PFS analysis of FA score in classical chordoma patients. (B) PFS analysis of FA score in chondroid chordoma patients. (C) OS analysis of FA score in classical chordoma patients. (D) OS analysis of FA score in chondroid chordoma patients. (E) PFS analysis of FA score in tumor volume $\leq 20,000 \mathrm{~mm}^{3}$ patients. (F) PFS analysis of FA score in tumor volume $>20,000 \mathrm{~mm}^{3}$ patients. (G) OS analysis of FA score in tumor volume $\leq 20,000 \mathrm{~mm}^{3}$ patients. (H) OS analysis of FA score in tumor volume $>20,000 \mathrm{~mm}^{3}$ patients.

Abbreviations: PFS, progression-free survival; OS, overall survival.

inflammation, and its association with tumor progression and patient prognosis has been identified in several cancers. ${ }^{26-29}$ However, to date, the value of fibrinogen in skull base chordoma patients has been unclear. Our results indicate that patients with high fibrinogen tend to have poor PFS and OS. The detailed mechanisms responsible for the prognostic role of fibrinogen remain poorly understood, and several studies may help explain our findings. Fibrinogen was thought to be an essential determinant of the metastasis ability of circulating tumor cells via the adhesion effect and survival of tumor cells rather than tumor stroma formation in a mouse model. ${ }^{30}$ Moreover, previous studies have indicated that fibrinogen could contribute to tumor angiogenesis by interacting with vascular endothelial cells and vascular endothelial growth factor, ${ }^{31}$ and fibrinogen could increase the migration and invasion potential of cancer cells via regulation of the 
Table 3 Univariable and Multivariable Cox Analysis of Factors Affecting PFS in Skull Base Chordoma

\begin{tabular}{|c|c|c|c|c|c|c|}
\hline \multirow[t]{2}{*}{ Variables } & \multicolumn{3}{|c|}{ Univariable Analysis } & \multicolumn{3}{|c|}{ Multivariable Analysis } \\
\hline & HR & $95 \% \mathrm{Cl}$ & $P$ value & HR & $95 \% \mathrm{Cl}$ & $P$ value \\
\hline Age (>55/ $\leq 55$ years) & 1.673 & $1.090-2.566$ & $0.018^{*}$ & NA & NA & 0.099 \\
\hline Sex (female/male) & 1.101 & $0.778-1.558$ & 0.587 & & & \\
\hline Tumor volume $\left(>20000 / \leq 20000 \mathrm{~mm}^{3}\right)$ & 1.678 & $1.178-2.392$ & $0.004 *$ & NA & NA & 0.228 \\
\hline Texture (hard or moderate/soft) & 1.674 & $1.115-2.512$ & $0.013^{*}$ & 2. 198 & $1.392-3.469$ & $0.00 I^{*}$ \\
\hline Blood supply (poor or moderate/abundant) & 0.690 & $0.480-0.992$ & $0.045^{*}$ & NA & NA & 0.190 \\
\hline Pathology (chondroid/classical) & 0.651 & $0.440-0.962$ & $0.031 *$ & 0.622 & $0.40 \mathrm{I}-0.964$ & $0.034^{*}$ \\
\hline Brainstem involvement (yes/no) & 1.241 & $0.864-1.782$ & 0.242 & & & \\
\hline Degree of resection (total/non-total resection) & 0.370 & $0.22 \mathrm{I}-0.620$ & $<0.00 I^{*}$ & 0.468 & $0.27 I-0.807$ & $0.006 *$ \\
\hline Postoperative radiotherapy (yes/no) & 1.423 & $0.985-2.057$ & 0.060 & NA & NA & 0.157 \\
\hline Fibrinogen (high/low) & 1.427 & $0.984-2.068$ & 0.061 & NA & NA & 0.518 \\
\hline Albumin (low/high) & 1.516 & $1.048-2.193$ & $0.027^{*}$ & NA & NA & 0.518 \\
\hline FA score & & & $0.017^{*}$ & & & $0.026 *$ \\
\hline 0 & I & reference & & 1 & reference & \\
\hline I & 1.593 & $1.101-2.303$ & $0.013^{*}$ & 1.656 & $1.105-2.482$ & $0.014^{*}$ \\
\hline 2 & 1.898 & $1.053-3.421$ & $0.033^{*}$ & 1.849 & $0.997-3.427$ & 0.051 \\
\hline
\end{tabular}

Note: *Indicate $p<0.05$.

Abbreviations: PFS, progression-free survival; $\mathrm{HR}$, hazard ratio; $\mathrm{Cl}$, confidence interval; NA, not acquired.

Table 4 Univariable and Multivariable Cox Analysis of Factors Affecting OS in Skull Base Chordoma

\begin{tabular}{|c|c|c|c|c|c|c|}
\hline \multirow[t]{2}{*}{ Variables } & \multicolumn{3}{|c|}{ Univariable Analysis } & \multicolumn{3}{|c|}{ Multivariable Analysis } \\
\hline & HR & $95 \% \mathrm{Cl}$ & $P$ value & HR & $95 \% \mathrm{Cl}$ & $P$ value \\
\hline Age (>55/ $\leq 55$ years) & 1.935 & $1.120-3.343$ & $0.018^{*}$ & NA & NA & 0.524 \\
\hline Sex (female/male) & $0.98 \mathrm{I}$ & $0.6|7-1.56|$ & 0.937 & & & \\
\hline Tumor volume $\left(>20000 / \leq 20000 \mathrm{~mm}^{3}\right)$ & 1.702 & $1.059-2.737$ & $0.028 *$ & NA & NA & 0.540 \\
\hline Texture (hard or moderate/soft) & 1.549 & $0.899-2.670$ & 0.115 & & & \\
\hline Blood supply (poor or moderate/abundant) & 0.527 & $0.317-0.876$ & $0.014 *$ & NA & NA & 0.386 \\
\hline Pathology (chondroid/classical) & 0.523 & $0.300-0.912$ & $0.022 *$ & NA & NA & 0.237 \\
\hline Brainstem involvement (yes/no) & 1.038 & $0.645-1.669$ & 0.878 & & & \\
\hline Degree of resection (total/non-total resection) & 0.321 & $0.147-0.701$ & $0.004^{*}$ & 0.419 & $0.188-0.930$ & $0.033^{*}$ \\
\hline Postoperative radiotherapy (yes/no) & 0.769 & $0.464-1.276$ & 0.309 & & & \\
\hline Recurrence (yes/no) & 9.192 & $3.352-25.205$ & $<0.001 *$ & 7.648 & $2.773-21.091$ & $<0.00 I^{*}$ \\
\hline Fibrinogen (high/low) & 1.583 & $0.978-2.562$ & 0.062 & NA & NA & 0.213 \\
\hline Albumin (low/high) & 2.218 & $1.383-3.558$ & $0.001 *$ & NA & NA & 0.213 \\
\hline FA score & & & $<0.001 *$ & & & $0.001 *$ \\
\hline 0 & 1 & reference & & I & reference & \\
\hline 1 & 1.633 & $0.983-2.715$ & 0.058 & 1.301 & $0.780-2.170$ & 0.314 \\
\hline 2 & 4.041 & $2.024-8.068$ & $<0.00 I^{*}$ & 3.959 & $1.960-7.995$ & $<0.001 *$ \\
\hline
\end{tabular}

Note: *Indicate $p<0.05$.

Abbreviations: OS, overall survival; $\mathrm{HR}$, hazard ratio; $\mathrm{Cl}$, confidence interval; $\mathrm{NA}$, not acquired.

epithelial-mesenchymal transition. ${ }^{32}$ Additionally, fibrinogen participates in the chronic inflammatory response by regulating the synthesis of interleukin-6 (IL-6) and altering the infiltration of leukocytes and lymphocytes. ${ }^{29,33}$ Another potential mechanism is that fibrinogen seems to block the contact between natural killer cells and tumor cells, thereby contributing to immune escape and weakening the eradication of tumor cells. ${ }^{34}$

Serum albumin, which is synthesized by the liver and is involved in inflammation, has been widely considered a useful tool for nutritional status assessment and serves as a valuable prognostic biomarker of disease progression and 

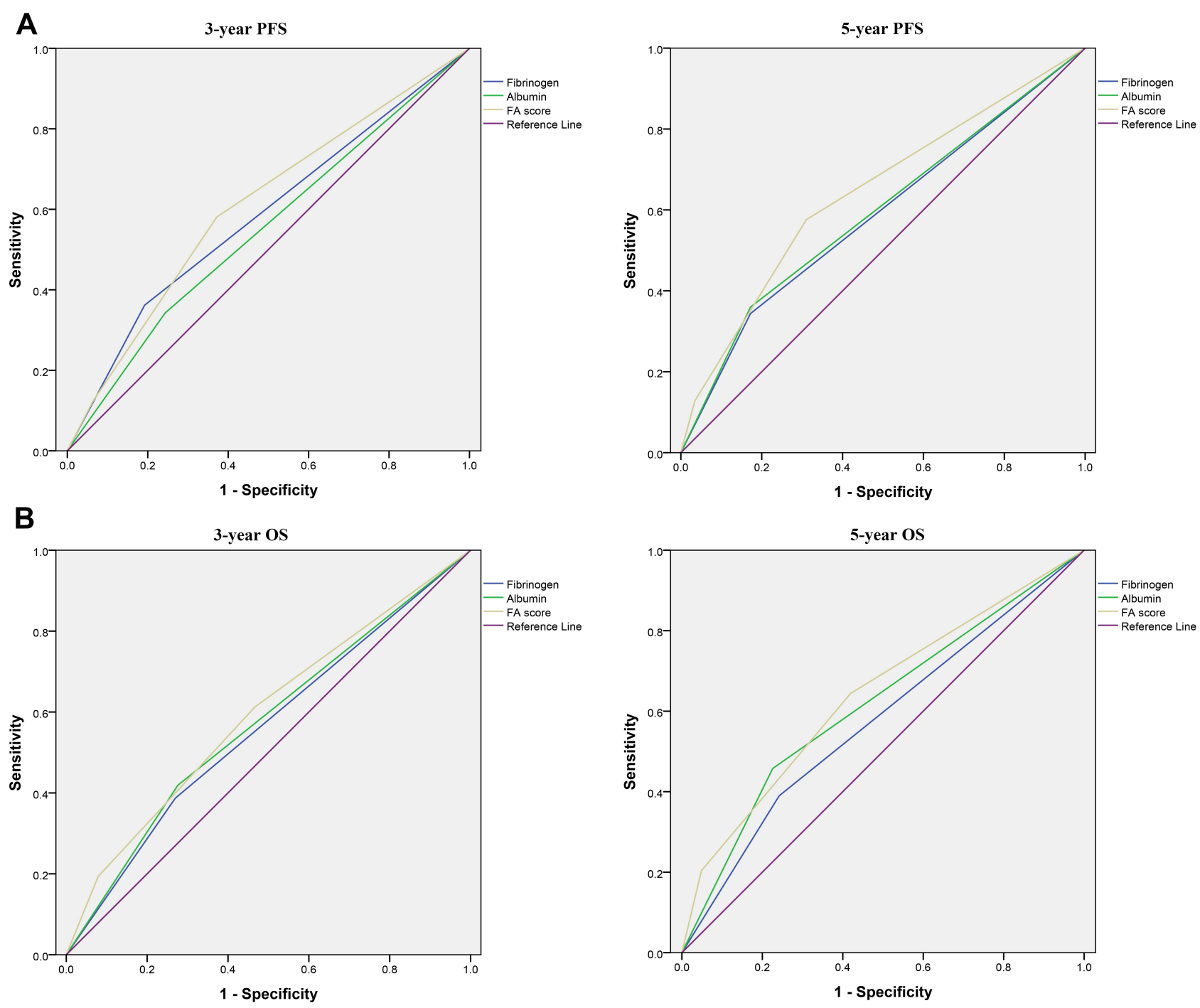

Figure 3 Comparison of the predictive performance of fibrinogen, albumin and FA score for 3-year and 5-year PFS, and 3-year and 5-year OS using ROC analysis. (A) 3-year PFS (left) and 5-year PFS (right). (B) 3-year OS (left) and 5-year OS (right).

Abbreviations: ROC, receiver operating characteristic; PFS, progression-free survival; OS, overall survival.

prognosis in various cancers. ${ }^{16}$ Moreover, malnutrition, which could be mirrored by a decreased albumin level, is associated with an increased likelihood of complications, such as infection, a poor human immune defence system, unfavorable response to therapy and a poor quality of life. ${ }^{15}$ Similarly, our results reveal that low levels of albumin are associated with a shorter PFS and OS in skull base chordoma patients. However, the reasons why albumin is a prognostic indicator in cancers and the mechanisms causing albumin to decrease remain poorly understood. Previous studies indicate that a low albumin level may be due to the release of several cytokines (such as IL-6 and tumor necrosis factor) by tumor cells during the process of the systemic inflammatory response. Additionally, IL-6 and tumor necrosis factor can be produced by hepatic macrophages in the presence of micrometastases in the liver. ${ }^{35}$ These cytokines contribute to the decrease in the serum albumin level by suppressing the hepatocyte synthesis of albumin and increasing the permeability of blood vessels. ${ }^{16}$ Considering the crucial relationships among inflammation, immunity and oncogenesis, ${ }^{7}$ exploring the role of albumin in inflammation and immunity may help reveal the deep mechanisms underlying the prognostic value of albumin in cancers. In addition, nutritional therapy aiming to improve cancer patient survival, such as albumin supplementation, merits further research.

The FA score, which is based on preoperative fibrinogen and albumin, was developed as a scoring system to reflect inflammation and the nutrition status by Matsuda and has been recognized as a novel clinical prognostic biomarker in several cancers, showing superior predictive 
power in esophageal cancer patients. ${ }^{19,20}$ Consistent with these findings, our results indicate that the FA score is an independent prognostic biomarker of PFS and OS in skull base chordoma patients. The FA score may be a valuable clinical score for the assessment of survival due to its relatively low cost and easy accessibility in daily practice.

Our study also has several limitations. The current study was a retrospective and single-centre research study with a small scale; therefore, selection bias may have played a role. Further prospective, multi-centre studies with large samples are required to confirm the cut-off points and the prognostic values of fibrinogen and albumin found in the current study. Additionally, the prognostic role of postoperative fibrinogen and albumin and the change between the preoperative and postoperative FA score were not analysed in our study. Moreover, further basic and clinical studies identifying how fibrinogen and albumin affect the prognosis of skull base chordoma patients are warranted.

\section{Conclusions}

Our data demonstrate that the preoperative level of albumin is associated with clinicopathological features and may predict outcomes in skull base chordoma patients. In particular, the FA score is an independent predictor of PFS and OS and has better predictive ability than fibrinogen or albumin alone. These results suggest that inflammation and nutrition may play crucial roles in the progression of skull base chordoma.

\section{Abbreviations}

PFS, progression-free survival; OS, overall survival; HR, hazard ratio; $\mathrm{CI}$, confidence interval; ROC, receiver operating characteristic; AUC, area under the curve.

\section{Data Sharing Statement}

The datasets analysed during the current study are available from Prof. Zhang on reasonable request.

\section{Ethics Approval and Informed Consent}

The current study protocol was approved by the ethics committee of Beijing Tiantan Hospital and carried out according to the 1964 Declaration of Helsinki. In addition, informed consent was obtained from all participants, and we further analysed the data anonymously.

\section{Acknowledgments}

We are grateful for the supporting of all patients.

\section{Funding}

This study was supported by the National Natural Science Foundation of China (81771489); Supported by Beijing Municipal Science \& Technology Commission (Z171100 000117002).

\section{Disclosure}

The authors report no conflicts of interest for this work.

\section{References}

1. Yu E, Koffer PP, DiPetrillo TA, Kinsella TJ. Incidence, treatment, and survival patterns for sacral chordoma in the United States, 1974-2011. Front Oncol. 2016;6:203. doi:10.3389/fonc.2016.00203

2. Tamborini E, Virdis E, Negri T, et al. Analysis of receptor tyrosine kinases (RTKs) and downstream pathways in chordomas. Neuro Oncol. 2010;12(8):776-789. doi:10.1093/neuonc/noq003

3. Zhai Y, Bai J, Wang S, et al. Differences in dural penetration of clival chordomas are associated with different prognosis and expression of platelet-derived growth factor receptor-beta. World Neurosurg. 2017;98:288-295. doi:10.1016/j.wneu.2016.07.096

4. Walcott BP, Nahed BV, Mohyeldin A, Coumans J-V, Kahle KT, Ferreira MJ. Chordoma: current concepts, management, and future directions. Lancet Oncol. 2012;13(2):e69-e76. doi:10.1016/S14702045(11)70337-0

5. Stacchiotti S, Sommer J. Building a global consensus approach to chordoma: a position paper from the medical and patient community. Lancet Oncol. 2015;16(2):e71-e83. doi:10.1016/S1470-2045(14)71190-8

6. Diakos CI, Charles KA, McMillan DC, Clarke SJ. Cancer-related inflammation and treatment effectiveness. Lancet Oncol. 2014;15 (11):e493-503. doi:10.1016/S1470-2045(14)70263-3

7. Grivennikov SI, Greten FR, Karin M. Immunity, inflammation, and cancer. Cell. 2010;140(6):883-899. doi:10.1016/j.cell.2010.01.025

8. Aggarwal BB, Vijayalekshmi RV, Sung B. Targeting inflammatory pathways for prevention and therapy of cancer: short-term friend, long-term foe. Clin Cancer Res. 2009;15(2):425-430. doi:10.1158/ 1078-0432.CCR-08-0149

9. Weisel JW. Fibrinogen and fibrin. Adv Protein Chem. 2005;70:247-299.

10. Kim KH, Park TY, Lee JY, et al. Prognostic significance of initial platelet counts and fibrinogen level in advanced non-small cell lung cancer. J Korean Med Sci. 2014;29(4):507-511. doi:10.3346/ jkms.2014.29.4.507

11. He Z-Q, Duan H, Ke C, et al. Evaluation of cumulative prognostic score based on pretreatment plasma fibrinogen and serum albumin levels in patients with newly diagnosed high-grade gliomas. Oncotarget. 2017;8(30):49605. doi:10.18632/oncotarget.17849

12. Tang L, Liu K, Wang J, Wang C, Zhao P, Liu J. High preoperative plasma fibrinogen levels are associated with distant metastases and impaired prognosis after curative resection in patients with colorectal cancer. J Surg Oncol. 2010;102(5):428-432. doi:10.1002/jso.21668

13. Du J, Zheng J-H, Chen X-S, et al. High preoperative plasma fibrinogen is an independent predictor of distant metastasis and poor prognosis in renal cell carcinoma. Int J Clin Oncol. 2013;18(3):517-523. doi:10.1007/s10147-012-0412-x

14. Fleck A, Hawker F, Wallace P, et al. Increased vascular permeability: a major cause of hypoalbuminaemia in disease and injury. Lancet. 1985;325(8432):781-784. doi:10.1016/S0140-6736(85)91447-3

15. von Meyenfeldt M. Cancer-associated malnutrition: an introduction. Eur J Oncol Nurs. 2005;9:S35-S38. doi:10.1016/j.ejon.2005.09.001

16. Gupta D, Lis CG. Pretreatment serum albumin as a predictor of cancer survival: a systematic review of the epidemiological literature. Nutr J. 2010;9(1):69. doi:10.1186/1475-2891-9-69 
17. Fiala O, Pesek M, Finek J, et al. Serum albumin is a strong predictor of survival in patients with advanced-stage non-small cell lung cancer treated with erlotinib. Neoplasma. 2016;63(3):471-476. doi:10.4149/ 318_151001N512

18. Liu Z-J, Ge X-L, Ai S-C, et al. Postoperative decrease of serum albumin predicts short-term complications in patients undergoing gastric cancer resection. World J Gastroenterol. 2017;23(27):4978. doi:10.3748/wjg.v23.i27.4978

19. Matsuda S, Takeuchi H, Kawakubo H, et al. Cumulative prognostic scores based on plasma fibrinogen and serum albumin levels in esophageal cancer patients treated with transthoracic esophagectomy: comparison with the Glasgow prognostic score. Ann Surg Oncol. 2015;22(1):302-310. doi:10.1245/s10434-014-3857-5

20. Cui J, Yu M, Zhang N, et al. Prognostic scores based on the preoperative plasma fibrinogen and serum albumin level as a prognostic factor in patients with upper urinary tract urothelial carcinoma. Oncotarget. 2017;8(40):68964. doi:10.18632/oncotarget.16483

21. Gui S, Zong X, Wang X, et al. Classification and surgical approaches for transnasal endoscopic skull base chordoma resection: a 6-year experience with 161 cases. Neurosurg Rev. 2016;39(2):321-323. doi:10.1007/s10143-015-0696-1

22. Hu B, Yang XR, Xu Y, et al. Systemic immune-inflammation index predicts prognosis of patients after curative resection for hepatocellular carcinoma. Clin Cancer Res. 2014;20(23):6212-6222. doi:10.1158/1078-0432.CCR-14-0442

23. Li M, Zhai Y, Bai J, et al. SNF5 as a prognostic factor in skull base chordoma. J Neurooncol. 2018;137(1):139-146. doi:10.1007/s11060017-2706-3

24. Zitvogel L, Tesniere A, Kroemer G. Cancer despite immunosurveillance: immunoselection and immunosubversion. Nat Rev Immunol. 2006;6(10):715-727. doi:10.1038/nri1936

25. Mantovani A, Allavena P, Sica A, Balkwill F. Cancer-related inflammation. Nature. 2008;454(7203):436-444. doi:10.1038/ nature 07205

26. Son HJ, Park JW, Chang HJ, et al. Preoperative plasma hyperfibrinogenemia is predictive of poor prognosis in patients with nonmetastatic colon cancer. Ann Surg Oncol. 2013;20(9):2908-2913. doi:10.1245/s10434-013-2968-8
27. Polterauer S, Grimm C, Seebacher V, et al. Plasma fibrinogen levels and prognosis in patients with ovarian cancer: a multicenter study. Oncologist. 2009;14(10):979-985. doi:10.1634/theoncologist.20090079

28. Zhao J, Zhao M, Jin B, et al. Tumor response and survival in patients with advanced non-small-cell lung cancer: the predictive value of chemotherapy-induced changes in fibrinogen. BMC Cancer. 2012;12:330. doi:10.1186/1471-2407-12-330

29. Jensen T, Kierulf P, Sandset PM, et al. Fibrinogen and fibrin induce synthesis of proinflammatory cytokines from isolated peripheral blood mononuclear cells. Thromb Haemost. 2007;97(5):822-829. doi:10.1160/TH07-01-0039

30. Palumbo JS, Kombrinck KW, Drew AF, et al. Fibrinogen is an important determinant of the metastatic potential of circulating tumor cells. Blood. 2000;96(10):3302-3309. doi:10.1182/blood. V96.10.3302

31. Staton CA, Brown NJ, Lewis CE. The role of fibrinogen and related fragments in tumour angiogenesis and metastasis. Expert Opin Biol Ther. 2003;3(7):1105-1120. doi:10.1517/14712598.3.7.1105

32. Shu YJ, Weng H, Bao RF, et al. Clinical and prognostic significance of preoperative plasma hyperfibrinogenemia in gallbladder cancer patients following surgical resection: a retrospective and in vitro study. BMC Cancer. 2014;14:566. doi:10.1186/1471-2407-14-566

33. Ridker PM, Howard CP, Walter V, et al. Effects of interleukin-1beta inhibition with canakinumab on hemoglobin A1c, lipids, C-reactive protein, interleukin-6, and fibrinogen: a phase IIb randomized, placebo-controlled trial. Circulation. 2012;126(23):2739-2748. doi:10.1161/CIRCULATIONAHA.112.122556

34. Palumbo JS, Talmage KE, Massari JV, et al. Platelets and fibrin(ogen) increase metastatic potential by impeding natural killer cell-mediated elimination of tumor cells. Blood. 2005;105(1):178-185. doi:10.1182/blood-2004-06-2272

35. McMillan DC, Watson WS, O'Gorman P, Preston T, Scott HR, McArdle CS. Albumin concentrations are primarily determined by the body cell mass and the systemic inflammatory response in cancer patients with weight loss. Nutr Cancer. 2001;39(2):210-213. doi:10.1207/S15327914nc392_8

\section{Publish your work in this journal}

OncoTargets and Therapy is an international, peer-reviewed, open access journal focusing on the pathological basis of all cancers, potential targets for therapy and treatment protocols employed to improve the management of cancer patients. The journal also focuses on the impact of management programs and new therapeutic agents and protocols on patient perspectives such as quality of life, adherence and satisfaction. The manuscript management system is completely online and includes a very quick and fair peer-review system, which is all easy to use. Visit http://www.dovepress.com/ testimonials.php to read real quotes from published authors. 